\title{
Postevent Information and Memory: Reply to Loftus, Schooler, and Wagenaar
}

\author{
Michael McCloskey \\ Johns Hopkins University
}

\author{
Maria Zaragoza \\ Kent State University
}

\begin{abstract}
In a recent article (McCloskey \& Zaragoza, 1985) we presented arguments and evidence in support of the contention that misleading postevent information does not impair memory for the original event. In their comment on our article, Loftus, Schooler, \& Wagenaar (1985) raise several objections to our conclusions. In this reply we consider these objections, suggesting that they present no serious challenges to our position. We also consider the implications of our arguments for forensic applications of postevent information research.
\end{abstract}

In a recent article (McCloskey \& Zaragoza, 1985) we made the following points in support of the contention that misleading postevent information has no effect on memory for the original event:

1. The procedures used in previous studies are inappropriate for assessing effects of misleading postevent information on memory for an event. Hence, the available data fail to demonstrate that misleading postevent information in any way impairs memory for the original event.

2. In six experiments using an appropriate procedure we found no effect of misleading postevent information on subjects' ability to remember the original event.

In their commentary on our article, Loftus, Schooler, and Wagenaar (1985) take issue with both of these points. In this reply we consider their objections, arguing that they present no serious challenges to our conclusion.

\section{Previous Postevent Information Research}

Central to the claim that misleading postevent information impairs memory for an event is a phenomenon obtained with what we have called the original test procedure. In a typical study (e.g., Loftus, Miller, \& Burns, 1978 ) subjects view a sequence of slides de-

We thank Howard Egeth, Sam Glucksberg, and Judith McKenna for their helpful comments.

Requests for reprints should be sent to Michael McCloskey, Department of Psychology, Johns Hopkins University, Baltimore, Maryland 21218. picting an event such as a robbery. Postevent information, such as a written narrative description of the event, is then presented. For subjects in the misled condition the narrative provides misleading information about a detail from the original event. For example, a hammer appearing in the slides might be described in the narrative as a screwdriver. For subjects in the control condition the narrative provides no specific information about the critical detail. After presentation of the postevent information, subjects are given a two-alternative forced-choice recognition test of memory for the original event. For the question about the critical detail, the alternatives are the item from the original slide sequence (e.g., hammer), and the item presented to misled subjects as misleading postevent information (e.g., screwdriver). Table 1 summarizes the design.

The consistent finding is that misled subjects perform more poorly than control subjects on the test question about the critical detail. This finding has been widely interpreted (e.g., Bekerian \& Bowers, 1983; Christiaansen \& Ochalek, 1983; Loftus, 1979a; Loftus \& Loftus, 1980; Loftus et al., 1978; Weinberg, Wadsworth, \& Baron, 1983) as evidence that misleading postevent information impairs memory for the original event, although researchers have debated whether original information (e.g., hammer) is lost from memory or is merely rendered inaccessible.

In our article we called the memory impairment interpretation into question, pointing out that there are two reasons to expect 
Table 1

The Original Test Procedure and the Modified Test Procedure

\begin{tabular}{lllll}
\hline Condition & Slides & Narrative & Original Test & Modified Test \\
\hline $\begin{array}{l}\text { Control } \\
\text { Misled }\end{array}$ & Hammer & - & Hammer versus Screwdriver & .Hammer versus Wrench \\
\hline
\end{tabular}

Note. From "Misleading Postevent Information and Memory for Events: Arguments and Evidence Against Memory Impairment Hypotheses" by M. McCloskey and M. Zaragoza, 1985, Journal of Experimental Psychology: General, 114, p. 5. Copyright 1985 by the American Psychological Association. Reprinted by permission.

poorer misled than control performance even if misleading postevent information has no effect on subjects' ability to remember the original event. First, misleading information will bias the responses of subjects who, for reasons unrelated to presentation of misleading information, do not remember what they originally saw. In the control condition subjects who do not remember the original information will guess on the two-alternative test question, and so should be correct $50 \%$ of the time. In the misled condition, however, expected performance for "don't-remember" subjects is less than $50 \%$ correct. Misled subjects who do not remember the original information (e.g., hammer), but do remember the misleading postevent information (e.g., screwdriver) will presumably choose the latter on the test, and so will systematically be incorrect. Thus, even if the proportion of subjects who remember the original information is the same in the misled condition as in the control condition, overall performance on the test will be lower in the misled condition. (See McCloskey \& Zaragoza, 1985, pp. 2-4, for a more complete explanation of this point.)

The second reason that poorer misled than control performance may occur even if misleading information does not affect memory for the original event is that some misled subjects who remember both the original information (e.g., hammer) and the misleading information (e.g., screwdriver) may choose the latter on the test, perhaps because they trust the information in the postevent narrative more than their own recollections of the original event.

We concluded that because of these two problems, the original test procedure is inappropriate for assessing effects of misleading information on memory for the original event-poorer misled than control performance is expected whether or not misleading information impairs memory for the event.

We also reviewed postevent information studies using other procedures (e.g., secondguess or warning procedures), arguing that because of logical or methodological flaws, these studies provide no basis for inferences about effects of misleading information on memory for the original event. Thus, we concluded that none of the available data demonstrate a memory impairment due to presentation of misleading postevent information.

Loftus et al. (1985) offer two responses to our conclusion. First, they argue that previous studies have uncovered many interesting empirical facts about the conditions under which subjects' reports are affected by misleading information. It is certainly true that subjects' reports may, at least under certain conditions, be affected by misleading postevent information. However, as we discuss in a later section, it is questionable whether this fact, in the absence of a theoretical interpretation, is important or even interesting.

Loftus et al. also argue that they have previously recognized the problems with the original test procedure, and have used a variety of other procedures to overcome these problems. In suggesting that the problems we noted had been recognized earlier, Loftus et al. are only partially correct; their claim that these problems were dealt with adequately is clearly incorrect. Consider first our argument that misleading information biases the responses of misled subjects who fail to remember the original information. To this argument Loftus et al. (1985, p. 376) reply that I.oftus et al. (1978) showed that "people are affected by misinformation even when it can be presumed that they would have otherwise 
spontaneously recalled the original information." This objection misses the mark. The Loftus et al. (1978) results do not imply that there is no response bias problem, but merely that this problem is not always the sole cause of misled/control differences. This point creates no problems for our position, because we have argued that the response bias is only one of two reasons to expect poorer misled than control performance even if misleading information has no effect on memory for the original event.

Turning now to the second of these reasons, Loftus et al. (1985) object to our suggestion that some subjects who remember both the original information (e.g., hammer) and the misleading information (e.g., screwdriver) may choose the latter on the test. This possibility, they assert, was considered in the Loftus et al. (1978) study, and is excluded by results obtained in that study. Once again, the objection does not stand up to scrutiny. Loftus et al. (1978) considered the possibility that the misleading information effect occurs because of misled subjects who remember both original and misleading information but choose the latter on the test in compliance with perceived demands in the experimental situation. Loftus et al. (1978) evaluated this demand characteristics interpretation by means of a debriefing procedure. ${ }^{1}$ Subjects, after taking the recognition test, completed a questionnaire asking them to report for the critical detail (a sign at an intersection) what they saw in the slides and what was mentioned in the postevent information. Only 2 of the 17 misled subjects who responded incorrectly on the recognition test correctly recalled both the critical detail from the slides (e.g., stop sign) and the misleading postevent information (e.g., yield sign). On the basis of this result, Loftus et al. (1985) dismiss our argument that some subjects who remember both original and misleading information may choose the latter on the test.

This conclusion is unwarranted, even if we ignore potential problems stemming from the social undesirability of admitting that one saw a stop sign in the slides yet chose a yield sign on the test. One important difference between the Loftus et al. (1978) demand characteristics interpretation and our remember-original-but-choose-misleading argument is that Loftus et al. proposed the former as a potential explanation for the entire misled/ control difference, whereas we offered the latter as only one of two reasons to expect a difference. Careful consideration of the Loftus et al. debriefing results reveals that these results pose no problem for our argument.

In the Loftus et al. (1978) experiment, 11 of the 30 control subjects and 17 of the 30 misled subjects responded incorrectly on the initial recognition test. Thus, presentation of misleading information led to incorrect responses by approximately 6 subjects who would have responded correctly had they not been misled. The debriefing results suggest that 2 subjects chose the incorrect response even though they remembered the original information. Hence, approximately one-third of the misled/control difference on the recognition test is attributable to subjects who remembered the original information yet chose the misleading information on the test. Clearly, this result is perfectly consistent with our argument that the misleading information effect may be due in part to subjects who remember both original and misleading information, and choose the latter on the test.

\section{The Modified Test Procedure}

We reported six experiments using a modified test procedure that, we argued, is appropriate for assessing effects of misleading post-

\footnotetext{
${ }^{1}$ The Loftus et al. (1978) and Loftus et al. (1985) use of the term demand characteristics is somewhat inappropriate. Applied to subjects who remember both original and misleading information yet choose the latter on the test, the term implies a deliberate choice of a response known to be incorrect in order to confirm the experimenter's hypothesis. However, subjects who remember the original information may choose the misleading information on the test for reasons not limited to a desire to conform to the experimenter's wishes. For example, a subject who thinks that the slides showed a hammer but remembers that the narrative described the tool as a screwdriver might reason that the experimenter who prepared the narrative must have known what was in the slides, and hence that the tool must have been a screwdriver. The demand characteristics label is also not apt when applied to deliberate compromise responses (see Loftus et al., 1985, p. 379); subjects may deliberately compromise for reasons other than a desire to conform to the experimenter's wishes. For example, subjects who think they saw a blue car but later are told that it was green may reason that the car was probably blue green.
} 
event information on memory for the original event. The modified procedure is the same as the original procedure except in the recognition test phase (see Table 1). In the modified procedure the item presented as misleading information (e.g., screwdriver) is not an option on the test; the test alternatives are the originally seen item (e.g., hammer) and a new item (e.g., wrench). With the modified procedure, poorer misled than control performance is expected if misleading information impairs memory for the original event. If, however, misleading information does not affect subjects' ability to remember the original information, no misled/control difference is expected. (See McCloskey \& Zaragoza, 1985, pp. 4-5, for a more detailed discussion of the modified test procedure.)

When we used the original test procedure, we obtained the usual misleading information effect. Performance averaged across the six experiments was $72 \%$ correct in the control condition and $37 \%$ correct in the misled condition. With the modified procedure, however, we found no misled/control difference. Across the six experiments performance was $75 \%$ correct in the control condition and $72 \%$ correct in the misled condition. We concluded that misleading postevent information does not impair memory for the original event. In the following sections we consider the two Loftus et al. objections to this conclusion.

\section{Sensitivity}

Loftus et al. argue that because a large proportion of subjects may choose the correct response on our two-alternative test by guessing, the test is insufficiently sensitive to detect small impairments in memory. Let us examine this argument. If a subject forgets the original information due to presentation of misleading postevent information, the subject's likelihood of responding correctly on the modified test is thereby reduced from $100 \%$ to $50 \%$. Consequently, with the modified test the expected misled/control difference is one-half the percentage of misled subjects who forgot the original information due to presentation of misleading information. For example, if misleading information causes forgetting of original information in $30 \%$ of the misled subjects, then a $15 \%$ misled/control difference is expected on the modified test.

Thus, the modified test is certainly not insensitive in the sense that expected performance remains constant as the percentage of subjects who remember the original information varies over some range; any change in the percentage of subjects who remember the original information is reflected in a change in expected performance on the test. Consequently, with a sufficient number of observations any arbitrarily small effect of misleading information could in principle be detected.

In this context it is important to note that we made very serious efforts to find an effect of misleading postevent information. We made within-subjects misled/control comparisons, and we used four critical items, so that each subject contributed two control and two misled observations. Further, we conducted not one but six experiments with the modified test, each involving between 60 and 84 subjects. Finally, after failing to find a significant misled/control difference in any of the six experiments, we conducted analyses on data pooled across experiments. In these analyses we made within-subjects comparisons involving data from 888 misled and 888 control test questions. Once again, we found no significant misled/control difference.

Loftus et al. (1985) cite an experiment by Benzing (1985) as evidence that when a more sensitive test is used, a small effect of misleading information on memory for the original event can be found. However, the experiment is seriously flawed, and does not demonstrate a memory impairment. The Benzing experiment differed from our modified test experiments in two ways. First, the recognition test questions listed four alternatives: the originally seen item and three new items not including the item presented as misleading information. Second, subjects responded not by choosing a single alternative, but rather by distributing 100 probability points among the four alternatives. For example, a subject reasonably confident of seeing a hammer might assign 70 points to the hammer alternative on the test, and 10 points to each of the other three alternatives. Loftus et al. argue that this "betting form" test is more sensitive than our modified test, both because 
the use of four alternatives reduces the likelihood of choosing correctly by guessing, and because the use of probability points "allows subjects to weight their responses according to how confident they are" (Loftus et al., 1985 , p. 377). Using the betting form procedure, Benzing found poorer misled than control performance.

There are two problems with the Benzing experiment, one conceptual and the other methodological. The conceptual problem is that, as Loftus et al. (1985) repeatedly emphasize, the dependent variable (i.e., the distribution of probability points) reflects not only what is remembered, but also confidence about what is remembered. The inclusion of confidence in the dependent measure is inappropriate, because the presentation of misleading information may well influence subjects' confidence even if it has no effect on their ability to remember (i.e., access stored representations of ) the original information. Consider, for example, a control subject and a misled subject who are equally able to remember seeing a hammer in the slide sequence. Suppose, however, that the misled subject also remembers that the postevent narrative referred to the tool in question as a screwdriver. The misled subject may as a result be less confident than the control subject that hammer is the correct item, and so may assign it fewer probability points on the betting form test, even when screwdriver is not an alternative on the test.

The methodological problem is one of incomplete counterbalancing. In our experiments three different versions of each critical item were used (e.g., hammer, wrench, and screwdriver for the tool critical item), and the assignment of these versions to the roles of original information, misleading information, and test foil was counterbalanced. The Benzing experiment used our stimulus materials and counterbalancing procedure, with the exception that two additional versions of each critical item (e.g., pliers and crowbar) were used as test foils to increase the number of test alternatives to four. These additional versions were used only as foils on the test, and never as original or misleading information. This failure to counterbalance fully leaves open the possibility that some misled

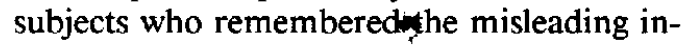

formation but not the original information were systematically biased toward incorrect responses on the test. Assume for the sake of argument that wrench is more similar to pliers than to hammer or screwdriver. Suppose now that hammer is the original information and wrench is the misleading information. On the test a misled subject who does not remember the original information, hammer, but does remember the misleading information, wrench, may well be biased toward pliers, the alternative most similar to the remembered item wrench. Because pliers was never used as original information, a response bias toward pliers is always a bias toward an incorrect response. Such a bias could lead to poorer misled than control performance, even if misleading information had no effect on memory for the original event.

Thus, the failure to counterbalance, and especially the use of a dependent measure that reflects confidence as well as memory for the original information, lead us to conclude that the Benzing results do not demonstrate that misleading information impairs memory for the original event.

\section{Memory blends}

Loftus et al.'s second objection to our modified test is that the test cannot detect memory blends (i.e., altered memory representations that include features of both the original and misleading information). Once again, the objection is not well-founded. First, the available data provide no clear evidence that memory blends actually occur. Studies (e.g., Loftus, 1977) in which some subjects gave blend responses (e.g., a response of blue green when blue is the original information and green is the misleading information) do not provide convincing evidence of blend memories. The blend responses may represent deliberate compromises between original and misleading information. Nor does the Weinberg et al. (1983) study emphasized by Loftus et al. demonstrate the occurrence of memory blends. The Weinberg et al. results can be interpreted without assuming that any of the subjects' responses, much less their memory representations, reflect a blending of original and misleading information (see McCloskey \& Zaragoza, 1985, p. 8). Finally, the Treisman 
work cited by Loftus et al. concerns illusory conjunctions in perception, and is irrelevant to the memory blend issue.

The second problem is that the memory blend notion is not sufficiently well-developed to merit serious consideration. Is the blend idea intended to replace the assertion that misleading information simply overwrites original information? If not, under what circumstances does each of the two types of memory alteration-overwriting and blending-take place? Further, how does memory blending occur? Why, when a stop sign and a yellow yield sign are blended, is the result a red yield sign as opposed to a yellow stop sign, or an orange sign with 5.5 sides and the word YSEOD on it? The Loftus et al. (1985) discussion seems to imply a highly intelligent blending process, one that would know to blend a stop sign and a yellow yield sign into a red yield sign, and would only produce a "hammerwrench" if such a tool did (or could plausibly?) exist. How would the process work? And what assumptions about memory in general would be required to provide a framework within which the process could fit?

We suggest, then, that the Loftus et al. (1985) sensitivity and memory blend comments do not pose serious problems for the conclusions we drew from our modified test results. We do, however, agree with Loftus et al. that the question of whether misleading postevent information affects memory for the original event is not settled. Our modified test results do not establish that misleading postevent information never impairs memory for the original event. However, because of the logical and methodological flaws in previous postevent information studies, our modified test results are at present the only findings bearing directly on the memory impairment issue.

\section{Forensic Applications}

The influence of postevent information research, and especially the memory impairment interpretation, have extended beyond basic issues in cognitive psychology. With increasing frequency, experimental psychologists are testifying as expert witnesses for the defense in criminal cases involving eyewitness identifications of the defendant. Often the expert asserts, among other things, that memory can easily be altered by information a witness encounters after the crime. However, our modified test results at the very least cast serious doubt on the memory alteration hypothesis. Hence, we suggest that there is insufficient scientific basis for expert testimony to the effect that postevent information may alter a witness's memory.

In line with the Loftus et al. suggestion that attention should be focused on subjects' reports and not their memories, it might be argued that a psychologist could quite properly offer expert testimony to the effect that postevent information may alter witnesses' responses (e.g., answers to questions, lineup identifications). This argument is not wellfounded, because there is insufficient basis for assuming that the results of the available studies can be generalized to situations involving eyewitnesses to crimes. The problem is not simply one of generalizing from the laboratory to the real world, but rather one of generalizing from any specific set of circumstances to any other set of circumstances. Before one can determine whether an effect obtained in one situation will generalize to another situation, one must know whether the differences between the situations are relevant or irrelevant to the occurrence of the effect. Our results, and the findings of some previous studies (e.g., Dodd \& Bradshaw, 1980; Loftus, 1979b), clearly indicate that even within the range of circumstances that have been studied, subjects' responses are not always affected by misleading information. At the least, the type of test, the type of original information, and the source of misleading information appear to be relevant in determining whether an effect is observed. Hence, the sweeping conclusion that misleading information always alters responses is unwarranted, and questions about the conditions under which responses are affected must be taken seriously. Answering these questions requires not, as Loftus et al. seem to suggest, a blind empirical exploration of various situations-one can never study all of the potentially relevant variations in circumstances. What is required instead is an understanding of the cognitive mechanisms underlying the phenomenon in question and, hence, of what variables are relevant or irrel- 
evant. At present, we have no such understanding; consequently, generalizations to situations involving eyewitnesses are essentially guesswork and as such should have no place in scientific expert testimony.

\section{References}

Bekerian, D. A., \& Bowers, J. M. (1983). Eyewitness testimony: Were we misled? Journal of Experimental Psychology: Learning, Memory, and Cognition, 9, 139145.

Benzing, W. (1985). Betting forms: A more sensitive measure of the impact of post-event information. Honors thesis, University of Washington, Seattle, Washington.

Christiaansen, R. E, \& Ochalek, K. (1983). Editing misleading information from memory: Evidence for the coexistence of original and postevent information. Memory \& Cognition, 11, 467-475.

Dodd, D. H., \& Bradshaw, J. M. (1980). Leading questions and memory: Pragmatic constraints. Journal of Verbal Learning and Verbal Memory, 19, 695-704.

Loftus, E. F. (1977). Shifting human color memory. Memory \& Cognition, 5, 696-699.
Loftus, E. F. (1979a). Eyewitness testimony. Cambridge, MA: Harvard University Press.

Loftus, E. F. (1979b). Reactions to blatantly contradictory information. Memory \& Cognition, 7. 368-374.

Loftus, E. F., \& Loftus, G. R. (1980). On the permanence of stored information in the human brain. American Psychologist, 35, 409-420.

Loftus, E. F., Miller, D. G., \& Burns, H. J. (1978). Semantic integration of verbal information into a visual memory. Journal of Experimental Psychology: Human Learning and Memory, 4, 19-31.

Loftus, E. F., Schooler, J. W., \& Wagenaar, W. (1985). The fate of memory: Comment on McCloskey \& Zaragoza. Journal of Experimental Psychology: General, 114, 375-380.

McCloskey, M., \& Zaragoza, M. (1985). Misleading postevent information and memory for events: Arguments and evidence against memory impairment hypotheses. Journal of Experimental Psychology: General, 114, 3-18.

Weinberg, H. I., Wadsworth, J., \& Baron, R. S. (1983). Demand and the impact of leading questions on eyewitness testimony. Memory \& Cognition, 11, 101104.

Received March 15, 1985

Revision received March 28, 1985

Improved Reproduction of Photomicrographs in Behavioral Neuroscience

Behavioral Neuroscience is pleased to announce new and improved photomicrograph reproduction. Previously, photomicrographs lacked the high resolution needed for detailed study. Beginning in 1985, the photomicrograph will appear twice: once in the text, as usual, and again in a special added signature of better quality, coated paper stock that will yield substantially more detail. 